\title{
En hommage à la lumière et à la noirceur
}

\author{
Catherine Cervin MD CCMF FCMF MAEd
}

D écembre est un mois compliqué. Beaucoup sont heureux de prendre une pause aux Fêtes. Pour d'autres, il s'agit d'une période triste, et plusieurs doivent travailler davantage pendant cette saison. Les journées sont plus courtes, plus sombres et plus froides, mais les décorations lumineuses apportent de la gaieté et de la chaleur.

Il y a plusieurs années, notre famille a commencé une tradition pour célébrer le solstice. Nous rendons hommage à la noirceur et à la lumière en cette nuit la plus longue et célébrons le retour progressif du soleil dans l'hémisphère Nord. Nous servons une fondue pâle (au fromage avec du pain baguette frais) pour le repas et une fondue foncée (au chocolat avec des fruits frais) pour le dessert. Nous nous assoyons en cercle, prenons un temps d'arrêt et réfléchissons à ce que nous voulons laisser derrière nous dans le noir et accueillir dans la lumière. Cette tradition est devenue un moyen d'échapper à la frénésie des Fêtes et une occasion de se réunir plus aisément qu'à Noël, que nous célébrons aussi.

Cette année fut difficile, marquée par l'incertitude et la tragédie, et éprouvante pour tant de Canadiens qui vivent dans des conditions précaires et dangereuses. Pourtant, en tant que médecins de famille, tous aux prises avec vos défis personnels, financiers ou familiaux, vous avez continué à prodiguer des soins à vos patients et à les soutenir pendant ces moments difficiles.

Pour moi, la cérémonie de remise de prix au Forum en médecine familiale est toujours un moment phare, encore plus en cette année sombre. J'espère que vous étiez nombreux à y assister virtuellement pour célébrer les réalisations de nos collègues et amis. Parmi les sujets forts qui ont été abordés, citons l'ardente défense des intérêts des peuples et communautés autochtones mal desservis (Catherine Cook, Mike Kirlew); la jeunesse, l'énergie et l'engagement envers l'éducation et la voix des patients et des communautés (Antoine Boivin, Nicole Stockley, Kim Lazare); la place centrale de la santé publique pour notre bien-être (Bonnie Henry, Jennifer Russell); et l'importance de l'urgence climatique et environnementale, éclipsée par la pandémie (Itua Iriogbe).

De nombreux lauréats nous ont rappelé la notion de service, dans le meilleur sens du terme:apporter sa contribution, être utile, travailler à un but. La pandémie a exposé et aggravé des problèmes et des inégalités de longue date. Les idées et citations qui suivent, provenant de nos lauréats, pourraient nous guider vers de meilleures structures, politiques et stratégies de sensibilisation.

- Catherine Cook, lauréate du Prix Calvin L. Gutkin de l'ambassadeur en médecine familiale, qui rend hommage à un chef de file dynamique de la médecine familiale canadienne qui s'est distingué par sa vision, son innovation et sa capacité à établir des relations efficaces: "L'accent mis sur la question de recherche est important d'abord pour les communautés [autochtones] plutôt que pour le chercheur seulement ${ }^{1}$."
- Antoine Boivin, médecin de famille et titulaire de la chaire de recherche du Canada sur le partenariat avec les patients et le public, qui a reçu le Prix Donald I. Rice pour sa contribution remarquable à l'enseignement, sa vision et son leadership dans la discipline de médecine de famille: "Les patients sont les experts de l'expérience de leur maladie, de leur situation et de leurs préférences [...] Leur participation aux comités d'élaboration des lignes directrices pourrait renforcer leur rôle dans la prise de décisions cliniques ${ }^{2}$."

- Mike Kirlew, qui a reçu le Prix Jean-Pierre Despins pour s'être illustré comme ardent défenseur et porte-parole public de la médecine familiale, des médecins de famille et de leurs patients: "C'est vraiment là que j'ai commencé à vouloir défendre leurs intérêts ${ }^{3}$ ", affirme-t-il en décrivant comment, dans des communautés autochtones accessibles uniquement par avion, il a vu des enfants sans médicaments contre l'asthme avoir du mal à respirer, et d'autres aux os fracturés gémir, car il ne disposait pas des moyens nécessaires pour atténuer leur douleur.

- George Carson, obstétricien-gynécologue nommé membre honoraire du CMFC pour avoir défendu systématiquement les soins de maternité et l'obstétrique rurale en médecine familiale: "En médecine, comme dans la vie, plus n'est pas toujours mieux. La meilleure médecine est fondée sur des données probantes, et les listes Choisir avec soin soulignent bien la façon dont nous pouvons continuer à améliorer la qualité de nos soins ${ }^{4}$."

- Sandy Buchman, récipiendaire du Prix W. Victor Johnston, remis à un médecin de famille reconnu sur la scène nationale ou internationale qui, par son leadership, a continuellement contribué à notre discipline, a consacré des heures de bénévolat pour aider à fournir des soins palliatifs aux sans-abri et a participé à des missions médicales en Afrique et en Amérique du Sud. Si vous avez besoin d'encouragement et d'inspiration en cette période sombre de l'année, veuillez consulter le site:https://fafm. cfpc.ca/fr/p-b/a-propos-des-prix-et-bourses/, où vous pourrez lire au sujet d'autres médecins de famille, cliniciens, éducateurs, chercheurs et leaders extraordinaires que je n'ai pas pu mentionner, faute d'espace.

Ces lauréats rappellent l'importance du travail des médecins de famille et du service que nous fournissons chaque jour à nos patients et à nos communautés. Je pense que chacun d'entre vous mérite un prix - prenez un moment pour songer à un patient pour qui vos soins ont été salutaires et à la différence que vous faites dans la communauté où vous travaillez.

En tant que présidente, je m'efforcerai d'entamer de nombreux dialogues avec vous (cathycervin@cfpc.ca). Qu'est-ce qui vous empêche de dormir la nuit? Qu'est-ce que vous célébrez et appréciez dans votre travail? Qu'est-ce que le Collège doit savoir et doit faire pour mieux vous servir? 